\title{
Transmission of Colicinogeny between Strains of Salmonella typhimurium Grown Together
}

\author{
By H. OZEKI*, B. A. D. STOCKER AND SYLVIA M. SMITH \\ Guinness-Lister Research Unit, Lister Institute of Preventive Medicine, \\ Chelsea Bridge Road, London, S.W. 1
}

(Received 17 November 1961)

\begin{abstract}
SUMMARY
Ability to produce colicines I, E1, E2, K or B was transferred to $S$ almonella typhimurium strain LT2 by growth in broth with suitable colicinogenic strains of Escherichia coli or Shigella sonnei. When LT2 (colI), i.e. carrying the colicine I factor, or LT2 $(\mathrm{colB})$ were grown overnight in broth with LT2 $\mathrm{col}^{-}$(non-colicinogenic), c. $50 \%$ of the latter became colicinogenic; LT2 (colE2) and LT2 (colK) did not transmit; LT2 (colE1) transmitted to only $c .0 \cdot 1 \%$ of the acceptor population. But LT2 carrying either $\operatorname{colI}$ or $\operatorname{colB}$ in addition to $\operatorname{colE2}$, colK or colE1, transmitted both factors.

When overnight broth cultures of LT2 (colI) and LT2 $\mathrm{col}^{-}$were mixed and incubated $c .40 \%$ of the latter acquired coll by $20 \mathrm{hr}$. (when the viable count had doubled); but only $c .0 \cdot 02 \%$ acquired $c o l I$ in $3 \mathrm{hr}$. The low initial transfer results from the fact that in a stock culture of LT2 (colI) only c. 1/5000 bacteria are 'competent donors', able to transmit colI. The later large increase in the proportion of colicinogenic bacteria probably results from 'epidemic spread' of the colI factor amongst the acceptor population, initiated by the few acceptor bacteria which originally receive it. It is supposed that most bacteria which have just acquired colI become competent donors. In a doubly colicinogenic strain most competent donors transmit both colicine factors.

Aeration by shaking during incubation interfered with transmission of colicinogeny, probably by abolishing the prolonged phase of slow growth of unaerated cultures. Growth in the presence of acriflavine did not 'cure' LT2 (colI) or LT2 (colI) (colE2) of colicinogeny, nor of ability to transmit.

LT2 (colE1) and LT2 (colE2) supported the epidemic spread of colI or colB about as well as did LT2 $\operatorname{col}^{-}$; but in LT2 (colK) the spread of $c o l I$ was greatly reduced and that of $c o l B$ somewhat reduced. The prior presence in an acceptor strain of one of the readily transmissible factors, colI or colB, did not interfere with the epidemic spread of the other. But LT2 (colI) did not become a competent donor on accepting colE2 and, by inference, coll from LT2 (colI) (colE2).
\end{abstract}

\section{INTRODUCTION}

Colicines are antibiotics produced by some strains of Enterobacteriaceae and active on others; colicinogeny, i.e. the ability to produce a colicine, is a stable heritable property of many Escherichia and Shigella strains (for review see Fredericq, 1957). It is a character of special interest to the bacterial geneticist; for

* Present address : Department of Chemistry, National Institute of Health, Shinagawa, Tokyo, Japan. 
some colicinogenic strains of Escherichia coli and Shigella sp. when grown in broth with non-colicinogenic strains transmit their colicinogenic property to some cells of the latter (Fredericq, 1954 $a$; Hamon, 1956). Colicinogeny could not be transmitted by culture filtrates. It is inferred that the character is transmitted from one bacterium to another by the transfer of a genetic determinant, during some kind of conjugation. We refer below to the postulated genetic determinant for colicinogeny as a colicine factor, indicating the colicine concerned by the symbol assigned to it by Fredericq. Thus; colicine factor I or, for short, colI, colE2, etc.

Experiments on the transmission of a colicine factor 'ER' (also termed ' K30', and falling in group E1; see Fredericq (1957)) between $\mathrm{F}^{+}$and $\mathrm{F}^{-}$sublines of Escherichia coli strain $\mathrm{K} 12$ showed that only $\mathrm{F}^{+}$strains transmitted colicinogeny; that all recombinants from crosses of $\mathrm{F}^{+}$non-colicinogenic and $\mathrm{F}^{-}$colicinogenic parents, and many from crosses of $\mathrm{F}^{+}$colicinogenic and $\mathrm{F}^{-}$non-colicinogenic parents, were colicinogenic; and that in the latter cross there was no detectable linkage between colicinogeny and any other character which was segregating (Fredericq \& Betz-Bareau, 1953 $a, b$; Fredericq, 1954b). It was inferred that colicinogeny resulted from possession of a non-chromosomal factor, transmissible during conjugation mediated by the $F$ agent. However, experiments on the inheritance of the same colicine factor ('K30') in crosses of $\boldsymbol{H} f \boldsymbol{r}$ with $\mathbf{F}^{-}$sublines of E. coli K12 (Alfoldi, Jacob \& Wollman, 1957; Alfoldi, Jacob, Wollman \& Maze, 1958) showed that the colicinogenic or non-colicinogenic character could be mapped at a particular point on the bacterial chromosome. Thus it seems that a colicine factor may be either located on the bacterial chromosome, being then transmissible only when the relevant portion of chromosome enters another bacterium; or may occupy some non-chromosomal site, and be transmissible by conjugation even in the absence of chromosomal transfer. Jacob \& Wollman (1958) defined as 'episomes' those non-essential heritable agents of bacteria which could be acquired or lost and having two alternative states; either on the chromosome, multiplying pari passu with it; or not on the chromosome, multiplying autonomously, sometimes more rapidly than the chromosome. The $F$ factor of $E$. coli, the colicine factor ' $\mathrm{K} 30$ ' of $\boldsymbol{E}$. coli and certain temperate phages (or prophages) of $\boldsymbol{E}$. coli were episomes as thus defined (Jacob, Schaeffer \& Wollman, 1960).

When the present work was begun, no genetic recombination by cell conjugation, mediated by the $F$ factor or otherwise, had been reported in Salmonella or between Salmonella and Escherichia (but see Miyake \& Demerec, 1959; Baron, Carey \& Spilman, 1959; Zinder, 1960). We therefore investigated the transmission of colicinogeny between Salmonella strains grown together, a phenomenon observed by Hamon \& Stocker (unpublished) and probably resulting from cell conjugation, hoping that fuller knowledge of this process would enable us to obtain recombination of the chromosomal genes of Salmonella by conjugation. This expectation has been realized (Ozeki \& Howarth, 1961; Smith \& Stocker, 1962; see also Stocker, 1960). In the present paper we describe the preparation of colicinogenic derivatives from nutritionally exacting sublines of Salmonella typhimurium strain LT2 and observations on the ability of such sublines to transmit their colicinogenic property to other LT2 sublines when the strains are grown together. Standard strains of Escherichia coli and Shigella sonnei producing known colicines, supplied by Professor P. Fredericq, were used as primary sources of various colicine factors; the process 
of transmission from strains of these genera to $S$. typhimurium has not been investigated in detail and, in particular, we have not tested the $F$ character of the donor strains.

We were for long unable to obtain transmission of colicinogeny to a substantial proportion of the cells of a non-colicinogenic LT2 strain by contact with a colicinogenic strain for short periods; the present paper therefore concerns results obtained when the colicinogenic and non-colicinogenic LT2 strains were incubated together in broth for some hours. We later found (Stocker, Smith \& Ozeki, in preparation) that Salmonella typhimurium cultures which have just acquired the ability to produce colicine I transmit this character to a high proportion of the bacteria of a non-colicinogenic strain in a short time, because a high proportion of the cells of such newly infected cultures can conjugate; we state this conclusion now because it helps in the interpretation of the results described below.

Salmonella typhimurium strain LT2 does not produce any detectable colicine; and it is resistant to all the colicines we have used, viz. I, E1, E2, B and K.

\section{METHODS}

Media. Nutrient broth was made from a tryptic digest of beef. The minimal medium contained: $\mathrm{K}_{2} \mathrm{HPO}_{4}, 10.5$ g.; $\mathrm{KH}_{2} \mathrm{PO}_{4}, 4.5 \mathrm{~g}$.; $\mathrm{MgSO}_{4}, 0.05 \mathrm{~g}$.; $\left(\mathrm{NH}_{4}\right)_{2} \mathrm{SO}_{4}$, $1 \mathrm{~g}$.; sodium citrate, $0 \cdot 47 \mathrm{~g}$.; glucose, $2 \mathrm{~g}$.; water, $1000 \mathrm{ml}$. The concentrations of nutritional supplements were as described by Lederberg (1950). Streptomycin was used at $1 \mathrm{mg} . / \mathrm{ml}$. These media contained $1.5 \%$ agar for plate culture, and $\mathbf{0 . 3 5} \%$ for soft agar. Bacterial strains were kept on Dorset egg slopes at laboratory temperature.

Bacterial strains. Table 1 a shows the strains of Escherichia coli and Shigella, colicinogenic for known type(s) of colicine, from which various colicinogenic properties were transferred to strains of Salmonella typhimurium.

Table $1 \mathrm{~b}$ lists the auxotrophic derivatives of Salmonella typhimurium strain LT2 used. A cysteine-exacting mutant, $c y s D-36$, was used in many experiments, because it is very stable, reversion to prototrophy having never been observed (Clowes, 1958). Mutants resistant to streptomycin were isolated from these stocks. Some naturally colicinogenic strains of $S$. typhimurium were also used. Among strains supplied by Dr E. S. Anderson, of the Enteric Reference Laboratory, Central Public Health Laboratory, Colindale Avenue, London, N.W. 9, 41 strains of 280 tested were found to be colicinogenic (Stocker, Ozeki \& Anderson, unpublished). Strains LT7 and LT22 (Zinder \& Lederberg, 1952), widely used in experiments on the genetics of $S$. typhimurium, were also observed to be colicinogenic, producing colicine I.

Table $1 c$ records the colicine-sensitive Escherichia coli strains, and their derivatives, used in testing for the production of colicines, i.e. colicine-indicator strains. For the most part these were colicine-resistant mutants of sensitive strains, either obtained from Professor P. Fredericq or isolated by us from colonies appearing in colicine inhibition zones. The $\mathbf{E}$ group of colicines are defined by the resistance to all $\mathrm{E}$ colicines and to phage $\mathrm{BF} 23$ of mutants obtained from sensitive strains by selection with any $\mathbf{E}$ colicine or with phage BF 23; within this group colicine E1 is recognized by the specific immunity to colicine $\mathbf{E} 1$ of strains made colicinogenic for colicine E1; and colicine E2 is similarly recognized by the specific immunity to 
colicine E2 of derivatives producing colicine E2 (Fredericq, 1956). We confirmed Fredericq's observation on the resistance pattern of $\boldsymbol{E}$. coli $\mathrm{K} 12$ lines made colicinogenic for E1 or E2, and used strain CL136, a K12 stock producing colicine E1, as an indicator sensitive to E2 but resistant to E1. We also confirmed Fredericq's observation that mutants selected for resistance to E1 are resistant to E2; but in stocks derived from $E$. coli strain $\phi$ of Gratia (1925) we found that mutants selected for resistance to E2 were still sensitive to E1, though less so than originally, and we used two such mutants as differential indicators.

\section{Table 1. Bacterial strains used}

(a) Standard colicinogenic strains, used as source of col factors : received from Professor P. Fredericq, Liège

\begin{tabular}{ll}
\multicolumn{1}{c}{ Strain } & \multicolumn{1}{c}{ Colicines } \\
produced
\end{tabular}

(b) Salmonella typhimurium strain LT2, auxotrophic mutants

Strain no.

$$
\begin{aligned}
& \text { cys } D-36 \\
& \text { cys } C-7 \\
& \text { ath } C-5
\end{aligned}
$$

Character

requires cysteine
requires cysteine
requires adenine and thiamine

Reference

Clowes (1958)
Clowes (1958)
Yura (1956)

Mutants of these strains resistant to streptomycin $(1 \mathrm{mg} . / \mathrm{ml}$.) are indicated by str- $r$ added to strain no. Colicinogenic derivatives are indicated by addition in parentheses of symbol for colicine

\begin{tabular}{|c|c|c|c|c|c|c|c|}
\hline \multirow{2}{*}{$\begin{array}{c}\text { Strain } \\
\text { no. }\end{array}$} & \multicolumn{5}{|c|}{ Sensitivity to colicine } & \multirow{2}{*}{$\begin{array}{l}\text { Streptomycin } \\
\text { sensitivity } \\
\text { (1 mg./ml.) }\end{array}$} & \multirow[b]{2}{*}{ Origin } \\
\hline & $\mathbf{I}$ & E 1 & E2 & $\mathbf{B}$ & $\mathbf{K}$ & & \\
\hline CL 18 & $\mathbf{S}$ & $\mathbf{S}$ & $\mathbf{S}$ & $\mathbf{S}$ & $\mathbf{S}$ & $\mathbf{S}$ & E. coli $\phi$ of Gratia (1925) \\
\hline CL 104 & $\mathbf{S}$ & $\mathbf{S}$ & $\mathbf{S}$ & $\mathbf{S}$ & $\mathbf{S}$ & $\mathbf{R}$ & $s t r-r$ mutant of CL 18 \\
\hline CL 147 & $\mathbf{R}$ & $\mathbf{S}$ & $\mathbf{S}$ & $\mathbf{r}$ & $\mathbf{S}$ & $\mathbf{S}$ & Colicine I-res. mutant of CL 18 \\
\hline CL 148 & $\mathbf{S}$ & $\mathbf{S}$ & $\mathbf{R}$ & $\mathbf{S}$ & $\mathbf{S}$ & $\mathbf{S}$ & Colicine E2-res. mutant of CL 18 \\
\hline CL150 & $\mathbf{R}$ & $\mathbf{S}$ & $\mathbf{S}$ & $\mathbf{r}$ & $\mathbf{S}$ & $\mathbf{R}$ & Colicine I-res. mutant of CL 104 \\
\hline CL 151 & $\mathbf{S}$ & $\mathbf{S}$ & $\mathbf{R}$ & $\mathbf{S}$ & $\mathbf{S}$ & $\mathbf{R}$ & Colicine E2-res. mutant of CL 104 \\
\hline CL152 & $\mathbf{R}$ & $\mathbf{S}$ & $\mathbf{R}$ & $\mathbf{r}$ & $\mathbf{S}$ & $\mathbf{R}$ & Colicine I-res. mutant of CL 151 \\
\hline CL 56 & $\mathbf{S}$ & $\mathbf{S}$ & $\mathbf{S}$ & $\mathbf{S}$ & $\mathbf{S}$ & $\mathbf{S}$ & E. coli K12-W677, thr, leu, thi \\
\hline CL 135 & $\mathbf{S}$ & $\mathbf{S}$ & $\mathbf{S}$ & $\mathbf{R}$ & $\mathbf{S}$ & $\mathbf{S}$ & E. coli K12-W1177/V* \\
\hline CL136 & $\mathbf{S}$ & $\mathbf{R}$ & $\mathbf{S}$ & $\mathbf{S}$ & $\mathbf{S}$ & $\mathbf{R}$ & E. coli K12-30*, carries colE1 \\
\hline CL.184 & $\mathbf{R}$ & $\mathbf{R}$ & $\mathbf{S}$ & $\mathbf{r}$ & $\mathbf{S}$ & $\mathbf{R}$ & Colicine I-res. mutant of CL 136 \\
\hline CL 185 & $\mathbf{S}$ & $\mathbf{R}$ & $\mathbf{R}$ & $\mathbf{S}$ & $\mathbf{S}$ & $\mathbf{R}$ & Colicine E2-res. mutant of CL 136 \\
\hline CL 142 & $\mathbf{S}$ & $\mathbf{S}$ & $\mathbf{S}$ & $\mathbf{S}$ & $\mathbf{S}$ & $\mathbf{R}$ & E. coli K12-Row* \\
\hline CL 143 & $\mathbf{R}$ & $\mathbf{S}$ & $\mathbf{S}$ & $\mathbf{S}$ & $\mathbf{S}$ & $\mathbf{R}$ & E. coli K12-Row/V* \\
\hline CL 144 & $\mathbf{r}$ & $\mathbf{S}$ & $\mathbf{S}$ & $\mathbf{R}$ & $\mathbf{S}$ & $\mathbf{R}$ & E. coli K12-Row/B* \\
\hline CL 145 & $\mathbf{S}$ & $\mathbf{R}$ & $\mathbf{R}$ & $\mathbf{S}$ & $\mathbf{S}$ & $\mathbf{R}$ & E. coli K12-Row/E* \\
\hline CL 146 & $\mathbf{S}$ & $\mathbf{S}$ & $\mathbf{S}$ & $\mathbf{S}$ & $\mathbf{R}$ & $\mathbf{S}$ & E. coli K12-Row/K* \\
\hline
\end{tabular}
agent concerned, e.g. cys $C-7(\operatorname{coll})$ indicates a derivative of strain $c y s C-7$ producing colicine $\mathrm{I}$.

(c) Colicine indicator strains

$\mathrm{S}=$ sensitive; $\mathrm{r}=$ partly resistant; $\mathbf{R}=$ completely resistant. $*=$ strains received from Professor P. Fredericq, of Liège, designated as shown. 
Culture methods. Cultures were grown at $37^{\circ}$. Broth cultures were usually grown, without aeration, in loosely capped containers; in some experiments $5 \mathrm{ml}$. cultures in $20 \mathrm{ml}$. bottles (loosely capped) were grown on a shaker (throw $9 \mathrm{~cm}$., 100 strokes/ min.).

Test for the production of colicine. To test for the production of colicine by colonies grown either from single bacteria or from stab inocula, the bacteria were killed by chloroform vapour and the plate covered with a layer of soft agar seeded with $c .10^{8}$ bacteria of an indicator strain; after overnight incubation colonies of colicinogenic bacteria were surrounded by clear zones. In experiments involving two or more colicines, the colicine or colicines produced were identified by the use of a set of test plates, each layered with a different indicator, resistant to one or more colicines.

\section{Preparation of colicinogenic derivatives of Salmonella typhimurium LT2 by contact with standard colicinogenic Escherichia and Shigella strains}

Sublines of strain LT2 producing each of the colicines I, B, E1, E2 or K, or combinations of them, were obtained by growing strain LT2 in mixed culture with appropriate 'donor' strains of Escherichia coli or Shigella sp. producing these colicines: $10 \mathrm{ml}$. broth was inoculated with about equal volumes $(0.1 \mathrm{ml}$; or a loopful) of broth cultures of the colicinogenic (donor) strain and a subline of strain LT2, generally one marked by nutritional requirements, etc. After overnight incubation, colicinogenic clones of the LT2 component of the culture were isolated, the procedure used varying according to the proportion of the LT2 bacteria which had acquired colicinogeny. When this proportion was high, the overnight mixed culture was streaked on nutrient agar and Salmonella colonies, recognizable by their smoothness, were picked and tested for colicinogeny. When the proportion was low the mixed culture was so treated that only the Salmonella component would grow when the mixture was plated; either the donor bacteria were killed by exposure to a suitable colicine (a chloroform-killed broth culture of a colicinogenic strain); or the mixture was plated on a medium, e.g. streptomycin agar, on which only the Salmonella strain could grow. Rare colicinogenic colonies amongst a large number of non-colicinogenic ones were then detected and isolated in one of the following ways: (i) Replica plates (Lederberg \& Lederberg, 1952) were tested for the presence of colicinogenic colonies; colonies corresponding to colicinogenic ones were then picked from the master plate. (ii) A first soft-agar layer inoculated with a suitable number of bacteria from the mixed culture was covered by a second uninoculated layer and after incubation a further layer containing indicator bacteria was added; after further incubation colicinogenic colonies underlying the centres of inhibition zones were picked (Fredericq, 1954a). (iii) When the proportion of Salmonella made colicinogenic was very small an inoculum of up to $10^{7}$ Salmonella was plated on a medium which would support only a very limited growth of the Salmonella, for instance unsupplemented minimal medium when the acceptor strain (cysD-36) required cysteine; after incubation the plate (not chloroformed) was covered with a layer of soft minimal agar supplemented with threonine, leucine and thiamine, and containing indicator bacteria (CL56) requiring these factors. After overnight incubation the indicator bacteria (but not the cysteine-exacting Salmonella) had grown and tiny inhibition zones were observed in the confluent growth; a small piece of agar containing a zone was cut out and suspended in broth, and from this (by 
repeating the same procedure, if necessary) the colicinogenic Salmonella clone could be isolated.

Singly colicinogenic derivatives of strain LT2. The standard colicine-producing strains used as donors of colicinogeny and the approximate proportion of LT2 cells which in a typical experiment became colicinogenic during overnight mixed culture are recorded in Table 2. Factors I and E2 were both obtained from Shigella sonnei strain P9, which produces both these colicines. After overnight culture of an LT2 subline with strain P9 about $50 \%$ of the LT2 cells had acquired colI, and about $5 \%$ coll and colE2 together, but no detectable proportion had acquired factor colE2 alone (see Ozeki \& Stocker, 1958). However, a subline of LT2 with the E2 factor of strain P9, but not the I factor, was obtained, apparently by the spontaneous loss of coll from an LT2 stock given colI and colE2 simultaneously from strain P9. It was later found that LT2 strains with factor E2 from strain P9 but without factor I could be regularly obtained either (i) by transduction, using phage PLT22 grown on an LT2 subline carrying colI and colE2 (Ozeki \& Stocker, 1958); or (ii) by interrupted mating of such a strain with LT2 $\mathrm{col}^{-}$; these results will be described in subsequent papers. Strain LT2 carrying colE2, but not colI derived from Sh. sonnei $\mathbf{P 9}$ behaved similarly, regardless of how it had been obtained.

Table 2. Transfer of colicine factors from standard colicinogenic strains of Escherichia coli and Shigella sonnei to sublines of Salmonella typhimurium strain LT2 by mixed culture

\begin{tabular}{|c|c|c|c|c|}
\hline \multicolumn{2}{|c|}{ Donor of colicinogeny } & \multicolumn{3}{|c|}{ Acceptor of colicinogeny } \\
\hline Strain & $\begin{array}{l}\text { Colicines } \\
\text { produced }\end{array}$ & Strain & Selected by & $\begin{array}{l}\text { Acceptor colonies } \\
\text { colicinogenic }\end{array}$ \\
\hline Shigella sonnei P9 & I \& E2 & LT2 cysD-36 str-r & Streptomycin & $\left\{\begin{array}{l}c .50 \%(\text { I only) } \\
c .5 \% \text { (I and E2) }\end{array}\right.$ \\
\hline E. coli K12-30 & E1 & LT2 cysD-36 & Colicine $\mathbf{K}$ & c. $10 \%$ \\
\hline E. coli $\mathrm{KJ7}$ & $\mathbf{B}$ & LT2 athC-5 str-r & Streptomycin & c. $20 \%$ \\
\hline E. coli $\mathbf{K 4 9}$ & $\mathbf{K}$ & LT2 $c y s C-7$ str-r & Streptomycin & c. $0.01 \%$ \\
\hline
\end{tabular}

$10 \mathrm{ml}$. broth was inoculated with loopfuls of broth cultures of the standard colicinogenic strain (donor) and of a subline of strain LT2 (acceptor). After overnight incubation the acceptor strain was selected and the proportion of its colonies which were colicinogenic was measured. Other LT2 sublines, and other methods of selection, have also been used, with similar results.

Doubly colicinogenic derivatives of strain LT2. To obtain doubly colicinogenic derivatives we used as acceptor strain an LT2 subline carrying one colicine factor, and incubated it in broth with a donor strain (Escherichia coli, Shigella sonnei or LT2) carrying some other factor. With one exception (Table 3c) singly colicinogenic LT2 sublines accept a second, different, colicine factor in the same way that noncolicinogenic LT2 does. LT2 (colI) (colE2), a doubly colicinogenic LT2 derivative used in many experiments, was prepared by several methods: (i) in a single step by growth in mixed culture with a donor strain carrying both factors; (ii) by transduction of colE2 by phage PLT22 from LT2 (colI) (colE2) into a stock already carrying colI; (iii) by transmitting colI by mixed culture to stocks carrying colE2 only, obtained either by transduction of colE2 into a non-colicinogenic stock, or by the spontaneous loss of colI from a stock carrying colI and colE2. 


\section{Test for transmission of colicine factors between LT2 sublines}

To test for the transmission of colicinogeny between LT2 sublines we used the technique described above, of overnight incubation of broth inoculated with equal numbers of bacteria of the donor and acceptor strains, in this case genetically labelled LT2 sublines. In most experiments we used a streptomycin-sensitive donor and a resistant acceptor, plated the mixed culture on streptomycin agar and tested for colicinogenic acceptor bacteria by the methods described above. In the case of colicine $\mathrm{K}$ no streptomycin-sensitive subline carrying colK was available for use as donor; but as the donor strain (cysC-7 str-r (colK)) was nutritionally exacting, LT2 wild-type was used as acceptor, and was selected by two serial passages of the mixed culture in liquid minimal medium.

\section{RESULTS}

The colicinogenic derivatives of Salmonella typhimurium LT2 obtained by contact with standard colicinogenic donor strains (see Methods) were indistinguishable from the strains from which they had acquired colicinogeny, in respect both of their range of activity and of the appearance of the inhibition zones they produced. That is, the colicine produced by the derivative seems to be identical with that produced by the original strain.

A method for detecting the production of colicine by individual bacteria of these colicinogenic LT2 sublines has been described elsewhere (Ozeki, Stocker \& de Margerie, 1959), together with observations on the proportion of the bacteria of such strains which liberated colicine, spontaneously or after ultraviolet irradiation. The colicinogenic LT2 derivatives varied in their stability. Sublines producing colicine I were stable, even on prolonged storage at room temperature on Dorset egg slopes; on a single occasion, however, a variant producing only colicine E2 was found in a stock initially producing both I and E2. Derivatives carrying colE1 or colE2 were also in general stable. Colicinogeny for colicines $\mathrm{B}$ and $\mathrm{K}$ was less stable; for when Dorset egg stock slopes of LT2 lines carrying colB or colK, kept at room temperature for a year or more, were streaked out, only 10-50\% and $c .0 \cdot 2 \%$, respectively, of the colonies were colicinogenic.

When a culture of an $\mathbf{F}^{+}$strain of Escherichia coli is incubated in a sub-lethal concentration of an acridine dye or a cobalt salt, many of the bacteria lose the $F$ factor and become $\mathbf{F}^{-}$(Hirota, 1956; Hirota \& Iijima, 1957). We therefore tested acriflavine for effect on the colI and colE2 agents in Salmonella typhimurium LT2. Strains cysD-36 (colI) and cysD-36 (colI) (colE2) were grown in broth with acriflavine, $80 \mu \mathrm{g} / \mathrm{ml}$; ; after $24 \mathrm{hr}$. at $37^{\circ}$ the count of viable bacteria had increased from c. $10^{6} / \mathrm{ml}$. to $c .7 \times 10^{7} / \mathrm{ml}$; ; all of several hundred colonies tested retained their colicinogenic character unchanged. All of 20 clones isolated from the acriflavinetreated cysD-36 (colI) culture transmitted colicinogeny in the same way as the untreated parent strain.

Singly colicinogenic LT2 sublines were tested for their ability to transmit colicinogeny to non-colicinogenic sublines during overnight mixed cultured in broth. Table $3 a$ records the results of a typical experiment; similar results were obtained when the donor or acceptor lines carried other genetic markers, and when other methods of selection of the acceptor strain were used. It appeared that in strain LT2 
colicine factors $\mathrm{I}$ and $\mathrm{B}$ are readily transmissible by mixed culture; that factors $\mathrm{E} 2$ and $\mathrm{K}$ are not transmitted to a detectable extent; and that factor $\mathrm{El}$ is transmitted at a very low rate. All of 20 'wild' colicinogenic strains of Salmonella typhimurium found to produce colicine I readily transmitted colicinogeny to LT2 $c y s D-36$ str-r; whereas none of 12 'wild' strains found to produce colicine E2 transmitted colicinogeny to a detectable extent.

Table 3. Transfer of colicine factors between LT2 strains during overnight mixed growth

(a) Singly colicinogenic donor and non-colicinogenic acceptor strains.

(b) Doubly colicinogenic donor and non-colicinogenic acceptor strains.

(c) Colicinogenic donor and colicinogenic acceptor strains.

State of acceptor bacteria after mixed growth

\section{Donor strain Acceptor strain}

(a) cys $D-36$ (colI) cys D-36 (colB) cysD-36 ( $\operatorname{col} E 1)$ cysD-36 (col E2) cysC-7 str-r (colK)

(b) cysD-36 (colI) (colB) cysD-36 str-r cysD-36 (colI) (colE1) cysD-36 str-r cysD-36 (colI) (colE2) cysD-36 str-r LT2w.t. †(colI)(colK) cysD-36 str-r

(c) athC-5 (colI)

ath $\mathrm{C}-5$ (colI)

ath $\mathrm{C}-5$ (coll)

athC-5 (coll)

ath $C-5($ colB $)$

ath $C-5(\operatorname{col} B)$

ath $C-5(\operatorname{col} B)$

ath $\mathrm{C}-5$ ( $\mathrm{colB})$

cysD-36 str-r cys $D-36$ str-r cys $D-36$ str $-r$ cysD-36 str-r LT2 (wild-type)

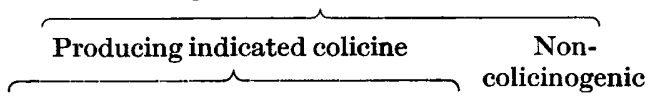

I, $50 \%$

B, $20 \%$

E1, 0.1\%

E2, $<0.001 \%$

*K, $<0.001 \%$

I, $\mathbf{3 . 5} \%$

I, $43 \%$

I, $55 \%$

I, $55 \%$

cysD-36 str-r (colB) I \& B, 33\%

athC-5 str-r (colE1) I \& E1, 50\%

cysC-7 str-r (colE2) I \& E2, $40 \%$

cysC-7 str-r (culK) I \& K, 0.4\%

cysC-7 str-r (colI) B \& I, 35\%

athC-5 str-r (colE1) $\quad \mathrm{B} \& \mathrm{E} 1,29 \%$

cysC-7 str-r (colE2) B \& E2, $58 \%$

cysC -7 str-r (colK)

B \& K, $8 \%$

B, 5.5\%
E1, 0\%
E2, 0\%
K, 0 \%
B, 67 \%
E1, 50\%
E2, 60\%
K, 99.6\%
I, 65\%
E1, 71 \%
E2, 42 \%
K, 92 \%

Loopfuls of overnight broth cultures of acceptor and donor strains were inoculated together into $10 \mathrm{ml}$. broth. After $18 \mathrm{hr}$. incubation the acceptor bacteria were selected on streptomycin agar and scored for colicinogeny with appropriate indicator strains.

* As the donor strain, cysC-7 str-r (colK) was streptomycin-resistant, but auxotrophic, the prototrophic acceptor bacteria were selected on minimal medium.

$\dagger$ Wild-type.

The $F$ agent can be transmitted by contact from $\mathrm{F}^{+}$strains of Escherichia coli to Salmonella typhimurium (Zinder, 1960); and one of our standard colicinogenic strains, $\boldsymbol{E}$. coli K12-30, used as source of the colE1 factor, was known to be $\mathrm{F}^{+}$(we have not investigated the $\boldsymbol{F}$ character of the other standard colicinogenic strains; see Table 2). We therefore considered the possibility that our colicinogenic derivatives of LT2 had become $\mathbf{F}^{+}$as well as colicinogenic. However, they did not show the kind of fertility found by Zinder (1960) in $\mathrm{F}^{+}$derivatives of LT2; and all were sensitive to phage SP6 which does not attack LT2 lines carrying the $F$ agent (Zinder, 1961). We conclude that none of our colicinogenic LT2 derivatives carries the $F$ agent.

Though factors E1, E2 and $\mathrm{K}$ are scarcely, if at all, transmitted by singly colicinogenic donor strains they are readily transmitted by donor strains which carry, 
in addition, one of the readily transmissible factors, I or B (see Table $3 b$ ). Most of the experiments described in this paper concern factors $I$ and $\mathbf{E 2}$, as representatives of the classes of factors which are, respectively, transmissible and non-transmissible by singly colicinogenic donor strains.

\section{Transfer of the coll factor}

When cysD-36 (colI) (donor) and cysD-36 str-rcol- (acceptor) were grown together in broth (inoculum $c .10^{5}$ bacteria/ml. of each strain), more than $50 \%$ of the acceptor population became colicinogenic during overnight incubation, by which time the total population was $c .10^{9}$ bacteria $/ \mathrm{ml}$. Undiluted broth cultures of the same donor and acceptor strains, grown overnight without aeration, were mixed in equal parts and re-incubated, without addition of fresh broth; after $3 \mathrm{hr}$. only c. $0.02 \%$ of the acceptor bacteria were colicinogenic, and after $6 \mathrm{hr} . c .0 \cdot 1 \%$; but after $20 \mathrm{hr}$. incubation the proportion of colicinogenic acceptor bacteria had increased to $c .50 \%$. No such transfer of colicinogeny occurred when the viable donor culture was replaced by either (1) a bacterium-free culture filtrate, or (2) the pasteurized $\left(60^{\circ}, 45 \mathrm{~min}\right.$.) supernatant of a centrifuged culture, or (3), a culture killed by shaking with chloroform. We conclude that transmission of colicinogeny between Salmonella typhimurium sublines grown together requires contact of 'live' cells, presumably because it occurs by conjugation, as has been inferred in other genera.

The transfer of colicinogeny during $20 \mathrm{hr}$. incubation of an undiluted mixture of unaerated overnight broth cultures of donor and acceptor strains might be thought to indicate that transmission of the colI factor was occurring under conditions where there was no bacterial growth. However, in our broth, incubated at $37^{\circ}$ without aeration by shaking, the growth of strain LT2 slows down when the count reaches $1-2 \times 10^{8} / \mathrm{ml}$, and after overnight incubation there are about $10^{9}$ viable bacteria/ ml.; slow growth continues on longer incubation, the number of viable bacteria increasing to $c .2 \times 10^{9} / \mathrm{ml}$. in a further $24 \mathrm{hr}$. Thus the transmission of colicinogeny to c. $50 \%$ of the acceptor bacteria took place in conditions permitting slow bacterial growth.

If a broth culture of strain LT2 is aerated by shaking during incubation the number of viable bacteria increases rapidly until it reaches $4-6 \times 10^{9} / \mathrm{ml}$. and then growth ceases. When aerated $18 \mathrm{hr}$. cultures of donor and acceptor strains were mixed, without dilution, very few of the acceptor bacteria became colicinogenic during $20 \mathrm{hr}$. further incubation, with or without shaking of the mixture. The almost complete absence of transmission of the colI factor under these conditions presumably results from the environmental conditions which are preventing bacterial growth (probably lack of any available energy source).

Shaking of a mixture of overnight unaerated cultures of donor and acceptor strains greatly reduced the proportion of acceptor bacteria which became colicinogenic during $20 \mathrm{hr}$. incubation; e.g. to $0.8 \%$, compared with $45 \%$ in an unshaken control. As stated above nearly all the acceptor bacteria which acquire colI in an unshaken mixture do so between the sixth and twentieth hour of incubation, during which time there is slow bacterial growth. In the shaken mixture by contrast growth would have ceased by the sixth hour of incubation and presumably no transmission of colicinogeny could occur thereafter. 
We therefore conclude that all the observed inhibitory effects of shaking on the transfer of colI can be accounted for by the effects of aeration by shaking on bacterial growth, without postulating any direct effect, e.g. on pair formation.

\section{Proportion of competent donors in overnight broth cultures of LT2 (colI)}

The very small proportion of acceptor bacteria which acquired the colI factor during the first few hours of incubation of a mixture of undiluted overnight broth cultures of donor and acceptor strains suggested that perhaps only a small fraction of the bacteria in a broth culture of an LT2 line carrying colI are 'competent donors', able to transmit colI (even though all bacteria in such a culture carry the factor). This hypothesis was tested, and the proportion of competent donors estimated, as follows: (i) graded numbers (e.g. 300, 3000 or 30,000) of bacteria of strain $c y s D-36$ (coll) in $0.2 \mathrm{ml}$. of saline were added to $0.2 \mathrm{ml}$. volumes of an overnight broth culture of a streptomycin-resistant acceptor strain; (ii) after either $15 \mathrm{~min}$. or $20 \mathrm{hr}$. incubation $10 \mathrm{ml}$. of streptomycin broth were added to each tube, to prevent further growth of the streptomycin-sensitive donor bacteria; (iii) after overnight incubation each tube was tested for the presence of colicinogenic bacteria of the streptomycin-resistant acceptor strain. In such an experiment (Table 4) the final test for

Table 4. Estimation of proportion of competent donors in a broth culture of strain cysD-36 (colI) by the dilution method

\begin{tabular}{|c|c|c|c|c|}
\hline \multirow{3}{*}{$\begin{array}{c}\text { No. of } \\
\text { bacteria of } \\
\text { donor strain } \\
\text { added }\end{array}$} & \multicolumn{4}{|c|}{$\begin{array}{l}\text { No. of tubes giving positive or negative } \\
\text { test for presence of streptomycin- } \\
\text { resistant colicinogenic bacteria } \\
\text { after incubation in streptomycin } \\
\text { broth added at }\end{array}$} \\
\hline & \multicolumn{2}{|c|}{$1.5 \mathrm{~min}$. } & \multicolumn{2}{|c|}{$20 \mathrm{hr}$. } \\
\hline & + & - & + & - \\
\hline $3 \times 10^{4}$ & 12 & 0 & 12 & 0 \\
\hline $3 \times 10^{3}$ & 4 & 8 & 5 & 7 \\
\hline $3 \times 10^{2}$ & 0 & 12 & 1 & 11 \\
\hline
\end{tabular}

$0.2 \mathrm{ml}$. volumes of saline dilutions of an overnight unshaken broth culture of the donor strain cysD-36 (colI) were mixed with $0.2 \mathrm{ml}$. volumes of a similar culture of strain $c y s D-36$ str-r, 24, tubes being set up for each size of inoculum. After $15 \mathrm{~min}$. incubation $10 \mathrm{ml}$. streptomycin broth was added to half the tubes of each set; the remainder were similarly treated after $20 \mathrm{hr}$. After $24 \mathrm{hr}$. at $37^{\circ}$ all tubes were streaked on streptomycin-agar plates, which after incubation were layered with a streptomycin-resistant indicator; reconstruction experiments showed that this final test would detect as few as $0.01 \%$ of streptomycin-resistant colicinogenic bacteria.

colicine production was either completely negative or strongly positive, the latter indicating that a large fraction of the acceptor bacteria had become colicinogenic; and the distribution of positive tubes was the same whether $15 \mathrm{~min}$. or $20 \mathrm{hr}$. had been allowed, without streptomycin, for transmission from the donor to the acceptor strain. We infer that positive tubes received, in the inoculum of donor bacteria, at least one competent donor bacterium, able to transmit colicinogeny, and that negative tubes received none. The mean number of effective donor bacteria inoculated per tube, calculated by the Poisson series from the proportion of negative tubes (15/24), is $c .0 \cdot 48$; as each tube received $c .3000$ bacteria the proportion of 
effective donors in the broth culture of strain $c y s D-36$ (colI) was $c$. 1/6000. The proportion of competent donors was also estimated by a different method. Samples of an overnight broth culture of a streptomycin-resistant acceptor strain were innoculated with graded numbers of bacteria of a streptomycin-sensitive donor strain. After $15 \mathrm{~min}$. at $37^{\circ}$, samples (c. $10^{6}$ acceptor bacteria/plate) were plated on streptomycin agar, and after incubation over-layered with a streptomycinresistant indicator. The number of colicine inhibition zones produced indicated the number of streptomycin-resistant acceptor bacteria made colicinogenic, and hence the number of effective donor bacteria. The calculated proportion of effective donor bacteria was $1 / 5000$ to $1 / 7800$ (Table 5).

Table 5. Estimation of proportion of competent donors in a broth culture of strain cysD-36 (colI) by plating method

No. of bacteria inoculated/plate

$\begin{array}{cccc}\begin{array}{c}\text { Donor strain } \\ \text { cys } D-36(\text { colI })\end{array} & \begin{array}{c}\text { Recipient strain } \\ \text { athC-5 str-r }\end{array} & \begin{array}{c}\text { No. of colicine } \\ \text { zones/plate* }\end{array} & \begin{array}{c}\text { No. of donor } \\ \text { bacteria/zone }\end{array} \\ 1.5 \times 10^{6} & \text { c } 10^{6} & 192 & 7 \cdot 8 \times 10^{3} \\ 3.2 \times 10^{5} & \text { c. } 10^{6} & 51 & 6.3 \times 10^{3} \\ 1.3 \times 10^{5} & \text { c. } 10^{6} & 24 & 5 \cdot 4 \times 10^{8} \\ 2.9 \times 10^{4} & \text { c. } 10^{6} & 4 & 7.3 \times 10^{3} \\ 1.5 \times 10^{4} & \text { c. } 10^{6} & 3 & 5 \cdot 0 \times 10^{3}\end{array}$

$1 \mathrm{ml}$. volumes of an overnight unshaken broth culture of the streptomycin-resistant recipient strain were seeded with graded numbers of bacteria from a similar culture of the streptomycinsensitive donor strain. After $15 \mathrm{~min}$. at $37^{\circ} 0.1 \mathrm{ml}$. volumes of $1 / 100$ dilutions were spread on streptomycin-agar plates; after $15 \mathrm{hr}$. incubation these were tested with a streptomycin-resistant indicator (CL 104).

* Average of three plates.

The large number of acceptor bacteria which on long incubation become colicinogenic, even in tubes receiving only one competent donor, do so, we believe, as a result of the serial transmission of the colI factor amongst the acceptor bacteria. To estimate the number of acceptor bacteria which become colicinogenic as a result of the inoculation of a single effective donor bacterium, graded numbers of bacteria of a donor strain were added to $1 \mathrm{ml}$. volumes of an overnight culture of a streptomycin-resistant acceptor strain and the tubes were re-incubated, without the addition of fresh broth; after $20 \mathrm{hr}$. (by which time there was only a c. twofold increase in the number of bacteria) the number of colicinogenic acceptor bacteria in each original tube was estimated by subculturing serial decimal dilutions of each tube in streptomycin broth and testing each subculture after incubation for the presence of streptomycin-resistant colicinogenic bacteria, as above. This experiment showed that original tubes which had received $c .3000$ bacteria of the donor strain contained, $20 \mathrm{hr}$. later either (i) $<10$ and presumably no colicinogenic acceptor bacteria or (ii) $>10^{5}$. The positive tubes had, by inference, received one (or a very few) effective donors. It is unlikely that the one effective donor in the inoculum had mated, successively, with $10^{5}$ acceptor bacteria during the $20 \mathrm{hr}$. incubation; the epidemic spread of the colI factor amongst the acceptor population seems the only plausible explanation of these results. 


\section{Transfer of the colE2, colK, colE1 and colB factors}

Singly colicinogenic LT2 sublines carrying either colE2 or colK did not transmit their colicinogenic properties to any detectable extent to non-colicinogenic LT2 during overnight growth in broth (Table $3 a$ ); transfer of colicinogeny to $1 / 10^{5}$ of the acceptor population would have been detected. LT2 sublines carrying only colE1 transmitted colicinogeny to $c . \mathbf{0 \cdot 1} \%$ of an acceptor population during overnight mixed culture. LT2 sublines carrying only colB transferred colicinogeny to $c .20 \%$ of an acceptor population during overnight mixed culture, we assume as a result of the epidemic spread of the $c o l B$ factor amongst the acceptor population.

Although singly colicinogenic LT2 (colE2) strains did not transmit colE2, overnight incubation of a doubly colicinogenic LT2 (colI) (colE2) strain with a noncolicinogenic acceptor resulted in the acquisition of both factors by some (e.g. $22 \%$ ) acceptor bacteria and of colI alone by a larger fraction (e.g. 55\%; see Table $3 b$ ); acceptor bacteria which had acquired colE2 only were not detected (cf. transmission of colI, and of colI and colE2 together, by Shigella sonnei P9 to LT2, Table 2). LT2 (colI) (colE2) stocks behaved alike in transmission tests, regardless of whether they had acquired the two factors simultaneously, or successively, in either order (see Strains, in 'Methods'). All the 12 'wild' Salmonella typhimurium strains producing colicine E2 transmitted colE2, with colI, after the latter factor had been introduced into them; a clone of Sh. sonnei P9 which had lost the transmitting ability of the doubly colicinogenic parent strain was found to have lost the ability to produce colicine I; ability to transmit was regained when factor colI was re-introduced.

Thus LT2 and several other strains of Salmonella typhimurium and Shigella sonnei strain P9 when carrying only colE2 do not transmit this factor, but when carrying both colE2 and colI transmit both factors.

The carriage of colI by a donor strain likewise resulted in some transmission of colK, not transmitted by singly colicinogenic strains, and facilitated the transmission of $\operatorname{colE} 1$ (Table $3 b$ ). In the case of the latter $c .5 \%$ of an acceptor population acquired colE1 (with colI) during overnight growth with a doubly colicinogenic donor, as against $c .0 \cdot 1 \%$ when the donor carried colE1 only.

From analogy with the transmission of colI we infer that most of the acceptor bacteria which acquire colE2 (or colK or colE1), together with colI, during overnight growth with a doubly colicinogenic donor strain do so through the epidemic spread amongst the acceptor population of the two factors in association. In an experiment similar to that recorded in Table 4 the proportion of competent donor cells in a broth culture of $c y s D-36$ (colI) (colE2) was measured; as in cysD-36 (colI) this proportion was only one in several thousand. In this experiment the tubes inferred to have received only one (or a very few) competent donor cells of cysD-36 (colI) (colE2) gave rise to streptomycin-resistant (acceptor) cultures producing colicine E2 as well as colicine $I$; this suggests that in the doubly colicinogenic strain all or nearly all competent donor cells can transmit both factors. If many acceptor bacteria which have just acquired both colI and colE2 (or colK or colE1) from such competent donors themselves become competent donors of both factors, epidemic spread of the two factors in association would be expected. The larger number of acceptor bacteria which acquire colI alone, instead of $\operatorname{colI}$ and $\operatorname{colE2}$, would be accounted for if during conjugation colI is sometimes transmitted without colE2. 
A few experiments indicated that the presence of $\mathrm{colB}$ in a donor strain facilitated the transfer of colE2, colK and colE1 in the same way that the presence of colI did, though not to quite the same extent.

As singly colicinogenic donor strains carrying either colI or colB transmitted colicinogeny to a high proportion of acceptor bacteria during overnight mixed culture (Table $3 a$ ), we could not use a comparison of the transmitting ability of singly and doubly colicinogenic donor strains as a test for the ability of one of these factors to assist the transfer of the other. However, when a donor strain carrying both $\operatorname{colI}$ and $\operatorname{colB}$ was incubated with a non-colicinogenic acceptor more than half the acceptor bacteria acquired both factors, while $36 \%$ acquired neither, a distribution incompatible with the hypothesis of independent transmission of the two factors (Table $3 b$ ). This suggests that competent donor bacteria carrying two transmissible factors resemble competent donors carrying one transmissible and one 'nontransmissible' factor, in that they usually transmit both factors.

\section{Transfer of colI and colB factors to colicinogenic acceptor strains}

When an acceptor strain already producing colicine E2 or E1 was incubated overnight with a donor strain producing colicine $\mathbf{I}$ (or B), a high proportion of the acceptor bacteria acquired the ability to produce colicine $I$ (or B), just as they did when the acceptor strain was non-colicinogenic (Table $3 c$ ); and the acquisition of colI or colB did not result in loss of the colicine factor already carried by the acceptor strain. When a 'donor' strain carrying colI was incubated with an 'acceptor' carrying $c o l B$, or vice versa, many bacteria of the 'acceptor' strain acquired the colicine factor carried by the 'donor', becoming doubly colicinogenic (Table 3c). Thus for most pairs of colicine factors tested the prior presence in an acceptor strain of one factor failed to prevent the epidemic spread, in this acceptor, of some other, transmissible, factor, introduced by a donor strain.

However, when an acceptor strain carrying colK was incubated overnight with LT2 (colI) or LT2 (colB) only $\mathbf{0 . 4}$ and $8 \%$, respectively, of the acceptor bacteria acquired colI or colB, as against $c .50 \%$ and $c .20 \%$, respectively, when the acceptor was $\mathrm{col}^{-}$. This limited spread of colI in LT2 (colK) has been observed in several experiments.

We could not directly test whether the prior presence of one coll factor in an acceptor strain would prevent the epidemic spread in this strain of some other colI factor, introduced by a donor strain, because all the colI agents we examined were indistinguishable. But as LT2 (colI) (colE2) transmits both factors to many bacteria of a non-colicinogenic acceptor strain (Table $3 b$ ), presumably through the epidemic spread of the two factors in association, we could test whether colE2 was transmitted in this way when the acceptor strain already carried colI. Strain $c y s D-36$ (colI) (colE2) was used as donor, and either $c y s D-36$ str-r col- or $c y s D-36$ str-r (colI) as acceptor; after overnight incubation of broth inoculated with $c .10^{7}$ bacteria $/ \mathrm{ml}$. of donor and of acceptor strains, $c .15 \%$ of the non-colicinogenic acceptor population, but no detectable proportion (and thus certainly less than $0.01 \%$ ) of the acceptor population carrying coll had acquired colE2. This inhibition by the prior presence of colI of the acquisition of colE2 from a donor carrying colI and colE2 suggests that there is no epidemic spread of colI and colE2 together in a population already carrying colI, and so presumably no spread of colI in such a population. 


\section{DISCUSSION}

The determinants for the production of five distinct colicines were thus transferred from Escherichia coli or Shigella sonnei into Salmonella typhimurium strain LT2, wherein each caused the production of a colicine indistinguishable by its spectrum of activity from that produced by the donor strains. Colicines E1 and E2, produced either by the original donor strains or by the LT2 derivatives, were distinguishable from each other not only by their lack of activity on indicator strains themselves producing the homologous colicine (Fredericq, 1956) but also by mutants resistant to colicine E2 but still sensitive to E1.

In the case of factors colI, colE1 and colE2 the newly introduced factors were never or almost never lost by the LT2 derivatives; but on storage at room temperature for many months non-colicinogenic bacteria become predominant in initially pure colicinogenic LT2 cultures carrying colK or colB. The reason for this difference in stability is unknown. Growth in the presence of acriflavine did not cause loss of colI or colE2. Perhaps the stability of these two factors in Salmonella typhimurium LT2 during storage and when exposed to acriflavine during growth results from their integration into the bacterial chromosome; for acriflavine treatment, though it 'cures' $\mathbf{F}^{+}$Escherichia coli strains of their autonomous $\mathbf{F}$ agent, does not affect the integrated $\boldsymbol{F}$ agent of $\boldsymbol{H} f r$ strains (Hirota, 1960).

The factors differed in their transmissibility by singly colicinogenic Salmonella typhimurium strains; colE2 and colK were not transmitted at all, colI and colB on prolonged incubation were acquired by a high proportion of the cells of a noncolicinogenic acceptor, and colE1 was of intermediate transmissibility. The ability of the source strain of Escherichia coli carrying the colK agent to transmit it to $S$. typhimurium may reflect an inherent difference in the behaviour of this factor in the two host species, or may result from the presence of some assisting factor, for instance the $F$ agent, in the colicinogenic $E$. coli strain.

Only a small fraction $\left(10^{-3}\right.$ to $\left.10^{-4}\right)$ of the bacteria in a broth culture of an LT2 strain carrying colI actually transmits the factor; and it appears that during long incubation of a mixed culture, colI spreads 'epidemically' in the acceptor population. The phenomenon of epidemic spread of colI (or colB) amongst noncolicinogenic populations can be explained by postulating that many or all bacteria newly infected by either (or both) these factors become 'effective donors', able to transmit. Experimental proof of the correctness of this postulate was later obtained (Stocker, Smith \& Ozeki, in preparation). Transmission to a high proportion of an acceptor population, and so, by inference, epidemic spread, took place only when conditions were such that there was slow bacterial growth, at high bacterial concentrations, for some hours. Aeration of mixed cultures interfered with transmission apparently because of the effect of aeration on the 'growth cycle' of a broth culture, rather than any direct effect on the ability of colicinogenic bacteria to transmit their agent. This effect of aeration is therefore probably unrelated to its effect in producing an $\mathrm{F}^{-}$(i.e. non-fertile) phenotype in $\mathrm{F}^{+}$strains of Escherichia coli $\mathrm{K} 12$ (Cavalli, Lederberg \& Lederberg, 1953).

The factors not transmitted at all by singly colicinogenic LT2 strains, viz. colE2 and $c o l K$, and the poorly transmissible colE1, were transmitted, with the transmissible factor concerned, to many acceptor bacteria by doubly colicinogenic donor 
strains carrying colI or colB as well as colE2, colK or colE1. These observations, and the data on the transmission of both their colicine factors by individual effective donors in doubly colicinogenic strains, suggest that when a bacterium becomes an effective donor, either spontaneously in an established colicinogenic strain carrying coll or colB or as a result of newly acquiring one of these factors, it transmits all the colicine factors it possesses.

During prolonged incubation the transmissible factor colI (or $\operatorname{colB}$ ) was acquired by a high proportion of the bacteria of acceptor strains already carrying some colicine factor other than that of the donor strain; except in the case where the donor carried colI and the acceptor colK (Table 3c). One may infer that, with this one exception, bacteria already carrying some other factor are susceptible to the epidemic spread of $\operatorname{colI}$ or $\operatorname{colB}$; and therefore that a bacterium carrying some other factor can accept colI or $\operatorname{colB}$ and then becomes an effective donor. On the other hand, colE2 was not transmitted from LT2 (colI) (colE2) to a high proportion of an acceptor carrying colI. One may probably infer that a bacterium already carrying colI does not become an effective donor when it accepts colE2, and presumably colI also. Thus a bacterium already carrying colI apparently does not become an effective donor when it acquires an additional colI factor.

The epidemic spread of colI initiated by a single effective donor may involve $>10^{5}$ acceptor bacteria, during a period in which the number of bacteria is only doubled. Under these conditions colI is clearly multiplying faster than the bacteria carrying it, and so may be said to be multiplying autonomously, by definition a property of an episome in its non-integrated state. By the same argument applied to epidemic spread initiated by doubly colicinogenic donors, factors colE2, colK and colE1 are likewise capable of autonomous multiplication, at a rate faster than their hosts. The present data do not show whether colicinogenic bacteria which are not effective donors (all bacteria in strains carrying only colE2 or colK; and nearly all in strains carrying colI or colB) are those in which the colicine factor(s) are integrated into the host chromosome; for their failure to transmit their factor(s) may result merely from failure to pair with acceptor bacteria.

The readily transmissible factors $\operatorname{colI}$ and $\operatorname{colB}$ in some ways resemble the $F$ factor of Escherichia coli. Thus the presence of colI or colB in LT2 enables it to transmit the otherwise non-transmissible colE2 and $\operatorname{colK}$; and $\mathrm{F}^{+}$but not $\mathrm{F}^{-}$ sublines of $E$. coli $\mathrm{K} 12$ transmit colicine factors $\mathrm{ER}(=\operatorname{colE} 1)$ and $\mathrm{S}_{2}$ (= colE2) (Fredericq, 1954b). Furthermore, under some conditions'the presence of the coll agent in a line of $S$. typhimurium results in conjugation and chromosomal recombination (Ozeki \& Howarth, 1961; Smith \& Stocker, 1962; see also Clowes, 1961). These similarities in behaviour of the colI agent in Salmonella typhimurium and the $\mathrm{F}$ agent in $E$. coli probably arise from the ability of each of these episomes to confer on its host the capacity to conjugate. It is likely that the large majority of the population carrying colI or colB are not 'competent donors' because the agent, though still present, does not confer ability to conjugate. It may be that the non-transmissibility (in the absence of assistance) of the colE2 and colK factors in S. typhimurium is due to their inability to confer this ability to conjugate on even a small fraction of bacteria carrying them. 
Most of the work described formed part of a London Ph.D. thesis of one of us (H.O.) We thank Professor P. Fredericq, and Dr N. D. Zinder for providing bacterial strains and phages.

\section{REFERENCES}

Alfoldi, L., Jacob, F. \& Wollman, E. L. (1957). Zygose létale dans des croisements entre souches colicinogènes et non-colicinogènes d'Escherichia coli. C.R. Acad. Sci., Paris, 244, 2974.

Alfoldi, L., Jacob, F., Wollman, E. L. \& Maze, R. (1958). Sur le déterminisme génétique de la colicinogénie. C.R. Acad. Sci., Paris, 246, 3531.

Baron, L. S., Carey, W. F. \& Spilman, W. M. (1959). Genetic recombination between Escherichia coli and Salmonella typhimurium. Proc. nat. Acad. Sci., Wash. 45, 976.

Cavalli, L. L., Lederberg, J. \& Iederberg, E. M. (1953). An infective factor controlling sex compatibility in Bacterium coli. J. gen. Microbiol. 8, 89.

Clowes, R. C. (1958). Investigation of the genetics of cysteineless mutants of Salmonella typhimurium by transduction. J. gen. Microbiol. 18, 154.

Clowes, R. C. (1961). Colicine factors as fertility factors in bacteria: Escherichia coli K-12. Nature, Lond. 190, 988.

FredericQ, P. (1954a). Transduction génétique des propriétés colicinogènes chez Escherichia coli et Shigella sonnei. C.R. Soc. Biol., Paris, 148, 399.

Frederica, P. (1954b). Intervention du facteur de polarité sexuelle $F$ dans la transduction des propriétés colicinogènes chez Escherichia coli. C.R. Soc. Biol., Paris, 148, 746.

FredericQ, P. (1956). Résistance et immunité aux colicines. C.R. Soc. Biol., Parts, 150, 1514.

FredericQ, P. (1957). Colicins. Annu. Rev. Microbiol. 11, 7.

Frederice, P. \& Betz-Bareau, M. (1953a). Transfert génétique de la propriété de produire un antibiotique. C.R. Soc. Biol., Paris, 147, 1653.

FredericQ, P. \& Betz-Bareau, M. (1953 $b$ ). Transfert génétique de la propriété colicinogène en rapport avec la polarité F des parents. C.R. Soc. Biol., Paris, 147, 2043.

Gratia, A. (1925). Sur un remarquable exemple d'antagonisme entre deux souches de Colibacille. C.R. Soc. Biol., Paris, 93, 1040.

Hamon, Y. (1956). Étude générale du transfert des propriétés colicinogènes. C.R. Acad. Sci., Paris, 242, 2064.

Hirota, Y. (1956). Artificial elimination of the $\mathrm{F}$ factor in Bact. coli K12. Nature, Lond. $178,92$.

Hirota, Y. (1960). The effect of acridine dyes on mating type factors in Escherichia coli. Proc. nat. Acad. Sci., Wash. 46, 57.

Hirota, Y. \& Irjima, T. (1957). Acriflavine as an effective agent for eliminating F-factor in Escherichia coli $\mathrm{K}-12$. Nature, Lond. 180, 655.

Jacob, F., Schafffer, P. \& Wollman, E. L. (1960). Episomic elements in bacteria. In Microbial Genetics, Symp. Soc. gen. Microbiol. 10, 67.

J ACOB, F. \& Wollman, E. L. (1958). Les episomes, éléments génétiques ajoutés. C.R. Acad. Sci., Paris, 247, 154.

LEDERBERG, J. (1950). Isolation and characterization of biochemical mutants of bacteria. Meth. Med. Res. 3, 5.

Lederberg, J. \& Lederberg, E. M. (1952). Replica plating and indirect selection of bacterial mutants. J. Bact. 63, 39!.

Mryake, T. \& Demerec, M. (1959). Salmonella-Escherichia hybrids. Nature, Lond. 183, 1586.

Ozeki, H. \& Howarth, S. (1961). Colicine factors as fertility factors in bacteria: Salmonella typhimurium strain LT2. Nature, Lond. 190, 986.

Ozeki, H. \& Stocker, B. A. D. (1958). Phage-mediated transduction of colicinogeny in Salmonella typhimurium. Heredity, 12, 525 (abstract).

Ozeki, H., Stocker, B. A. D. \& DE MArgerie, H. (1959). Production of colicine by single bacteria. Nature, Lond. 184, 337. 
Sмiтh, S. M. \& Stocker, B. A. D. (1962). Colicinogeny and recombination. Brit. med. Bull. 18, 4.6.

Stocker, B. A. D. (1960). Introduction : micro-organisms in genetics. In Microbial Genetics, Symp. Soc. gen. Microbiol. 10, 1.

YURA, T. (1956). Evidence of non-identical alleles in purine-requiring mutants of Salmonella typhimurium. Publ. Carneg. Instn, 612, 63.

ZiNDER, N. D. (1960). Sexuality and mating in Salmonella. Science, 131, 924.

Zinder, N. D. (1961). A bacteriophage specific for F - Salmonella strains. Science, 133, 2069.

Zinder, N. D. \& Lederberg, J. (1952). Genetic exchange in Salmonella. J. Bact. 64, 679. 
